\title{
Pharmacobezoar: An Unusual Cause of Large Bowel Obstruction
}

\author{
Rishitha Yelisettia, b, Areig Awad ${ }^{\mathrm{a}}$, Bushra Ambreen ${ }^{\mathrm{a}}$, Ritika Zijoo, \\ Sara Wallach ${ }^{\mathrm{a}}$
}

\begin{abstract}
Large bowel obstruction (LBO) is an abdominal emergency with high morbidity and mortality rates if left untreated. LBO is four to five times less frequent than small bowel obstruction (SBO) and the causes of LBO and SBO differ substantially. Colonic malignancy remains the most common cause of LBO (>60\%). Additional causes of LBO include entities such as diverticulitis, colonic volvulus, and adhesion. Herein we present a case of acute LBO caused by pharmacobezoar.
\end{abstract}

Keywords: Pharmacobezoar; Large bowel obstruction; Polypharmacy

\section{Introduction}

A bezoar is defined as a foreign body resulting from accumulation of ingested material, commonly found as a hard mass or concretion in the gastrointestinal (GI) tract. Pharmacobezoars are composed of ingested medications. Formation of an intestinal pharmacobezoar is a rare condition. It develops in the GI tract typically due to alterations in anatomical structure and/ or intestinal motility. It may form after intake of various oral pharmaceutical preparations of drugs, both as a result of an acute overdose and through chronic use of therapeutic doses of a drug. Large bowel obstruction (LBO) secondary to pharmacobezoar is a rare phenomenon. We present an unusual case of LBO due to pharmacobezoar which was treated endoscopically.

\section{Case Report}

The patient, a 81-year-old Caucasian male with past medical

Manuscript submitted May 24, 2017, accepted June 13, 2017

aDepartment of Internal Medicine, Saint Francis Medical Center, Seton Hall University, Trenton, NJ, USA

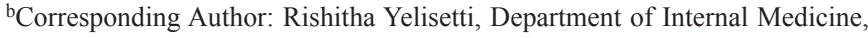
Saint Francis Medical Center, Seton Hall University, Trenton, NJ, USA. Email: ryelisetti@stfrancismedical.org

doi: https://doi.org/10.14740/gr871w history of chronic obstructive disease, coronary artery disease, subarachnoid hemorrhage secondary to a fall about 3 years ago, benign prostatic hypertrophy, balder diverticula, sick sinus syndrome with a pacemaker placement, ischemic cardiomyopathy, atrial fibrillation, hypertension, gastroesophageal reflux disease, and anxiety disorder, presented to the emergency department with complaints of gradually worsening generalized abdominal pain that was dull and crampy in nature, $7-8 / 10$ intensity since the last 2 days. Pain was intermittent and was aggravated by moving and consuming food, partially relived with rest and over-the-counter pain analgesics. It was associated with abdominal distention, nausea and one episode of non-bloody, non-bilious vomiting. He did not notice any changes in his bowel habits. He denied any sick contacts or recent history of travel. His last bowel movement was 2 days prior to the presentation. He noted he was not able to pass gas since then as well. He denied any hematochezia, melena or hematemesis. He denied any dysuria, fevers, chest pain, and shortness of breath or palpitations. Patient reported that for the last 2 months, he was having unintentional weight loss but failed to quantify it. Patient reported that his last colonoscopy was about 8 years ago and was normal. Past surgical history was significant for laparoscopic cholecystectomy and cervical laminectomy. Family history was pertinent for colon cancer in mother and brother, and diabetes in father. Patient was a resident of a nursing home and he denied active tobacco, alcohol or illegal drug use. He was allergic to tetracyclines. Patient's home medications included: Advair Diskus - 250/50 $\mu \mathrm{g}$ - one puff by inhalation twice daily; amiodarone $200 \mathrm{mg}$ daily; warfarin $7.5 \mathrm{mg}$ daily; escitalopram $20 \mathrm{mg}$ once daily; iron sulfate (Feosol) $325 \mathrm{mg}$ daily; furosemide $20 \mathrm{mg}$ once daily; potassium chloride $10 \mathrm{mEq}$ daily; Mucinex (Guafenesin) $600 \mathrm{mg}$ twice daily; Montelukast $10 \mathrm{mg}$ once daily; bupropion $15 \mathrm{mg}$ daily; cetrizine $10 \mathrm{mg}$ daily; over-the-counter multi-vitamin tablets - one daily; and over-the-counter vitamin $\mathrm{C}$ tablet once daily.

Review of systems was positive for unquantified weight loss, anorexia, constipation, abdominal discomfort, and bilateral leg swelling. On physical examination, vitals at the time of presentation to the emergency department showed temperature of $98.1^{\circ} \mathrm{F}\left(36.7^{\circ} \mathrm{C}\right)$, respiratory rate of 19 breaths/min, regular, unlabored, pulse rate of 67 beats $/ \mathrm{min}$, regular rate and rhythm, blood pressure of $118 / 62 \mathrm{~mm} \mathrm{Hg}$, and oxygen saturation of $100 \%$ on room air. Patient was an alert, oriented male who was anxious and in some distress. There was no jaundice. 
His conjunctivae and eyelids were normal. He had full extraocular movements. His oral cavity showed dry oral mucosa. There was no evidence of adenopathy in his head, neck or supraclavicular regions. Trachea was midline. Thyroid was not enlarged. The lungs fields were clear, symmetrical air entry. His heart exam was normal without murmurs, rubs or bruits. Abdominal examination revealed a distended, form abdomen with generalized tenderness on palpation. Percussion revealed a tympanic note. Examination was negative for guarding, rebound tenderness, organomegaly, shifting dullness, Rovsing's sign, Murphy's sign, and costo-vertebral angle tenderness. Hyperactive bowel sounds were appreciated in all the four quadrants. Patient refused a rectal examination. Neurological examination was normal. There was $1+$ pitting pedal edema in the bilateral lower extremities. Computerized tomography (CT) of the abdomen was performed. It showed apparent transition zone at the rectosigmoid junction with marked dilatation of the large bowel proximal, elevated left hemi-diaphragm with interposition of large bowel underneath and signs of compressive atelectasis of the left upper lobe. Bladder stones were evident in a right-sided bladder diverticulum. The small bowel was not dilated. Patient was admitted to the general medical floor with a provisional diagnosis of bowel obstruction, ruling out colorectal carcinoma. Advanced imaging with a CT scan further solidified the suspicion of bowel obstruction and probable mass. Surgery and gastroenterology consultations were requested. The initial plan was to get a colonoscopy or a sigmoidoscopy to further characterize any suspected mass and then proceed with a colectomy - Hartmann procedure with a diverting colostomy. Patient was put nil per oral and supportive intravenous hydration was provided. A colonoscopy was ordered in the anticipation of a colonoscopy. Patient however did not have any bowel movement after this. A decision was taken to proceed to colonoscopy anyway. Colonoscopy revealed partially dissolved pills that were clumped together in the sigmoid colon with a questionable component of sigmoid volvulus. Pill bezoar was removed carefully and on visual inspection demonstrated multiple aggregated pills that were causing obstruction. Additional inspection of the colon was normal with no ulceration, masses or polyps. Patient's LBO resolved completely after this and he recovered well tolerating diet with no additional symptoms.

\section{Discussion}

Pharmacobezoars are a rare kind of bezoars caused by accumulation of medications or medication vehicles in GI tract. Fiber containing laxatives and extended release drugs are usual culprits. However enteric coated tablets can also form bezoars because of insolubility of carrying vehicle. Decreased intestinal motility is an important risk factor for formation of pharmacobezoars. Most cases of bezoars are reported in patients with diminished gastric motility or previous GI surgeries. Other factors that may contribute to formation of pharmacobezoars include diminished gastric secretion, autonomic dysfunction in diabetes mellitus and myotonic dystrophy [1]. Bezoars are an uncommon cause of intestinal obstruction accounting for about $0.4-4 \%$ of cases. Stomach is the most common site for obstruction from bezoars followed by small intestines. Bedioui et al described a case series of 15 patients with small bowel obstruction (SBO) secondary to bezoars [2]. Colonic obstruction secondary to bezoars is a rare phenomenon. Law et al described a case of colonic obstruction secondary to phytobezoars which was treated with urgent laparotomy secondary to peritonitis [3]. Few other cases of colonic obstruction secondary to phytobezors are also reported. Medication bezoar leading to colonic obstruction is extremely rare. Agha et al in 1984 presented a case of colonic obstruction secondary to psyllium. A case report of ileocecal obstruction secondary to antacids has also been reported $[4,5]$. We did not come across any case report of LBO secondary to non-laxative medications. Most patients with colonic bezoar present with vague abdominal pain, nausea and vomiting which may progress to intestinal obstruction. Further complications may arise if not treated promptly and include perforation, peritonitis or bowel ischemia and necrosis. CT scan may help to diagnose bezoar. Sonographic presentation typical of bezoar is intraluminal mass with mottled gas pattern [6]. Treatment of colon obstruction due to bezoar depends upon location, size, and nature of bezoar and associated complications. Conservative management is mainstay of treatment for uncomplicated bowel obstruction. Conservative management includes manual disimpassion and enemas. Colonoscopic removal of bezoar can be considered after failed conservative management. Surgery is reserved for patients with failed conservative management or complications. Colon obstruction secondary to pharmacobezoar is rare and challenging diagnosis. Very few such case reports have been published. During our research, we did not come across any case report of LBO due to non-laxative medications.

\section{Grant Support}

None.

\section{Financial Disclosures}

None.

\section{Conflicts of Interest}

None.

\section{References}

1. Park JW, Park JG, Kwon OK, Lee KU, Hong SC, Choe $\mathrm{KJ}$, et al. Clinical analysis of Bezoars. Korean J Gastroenterol. 1991;23:32-38.

2. Bedioui H, Daghfous A, Ayadi M, Noomen R, Chebbi F, Rebai W, Makni A, et al. A report of 15 cases of smallbowel obstruction secondary to phytobezoars: predisposing factors and diagnostic difficulties. Gastroenterol Clin 
Biol. 2008;32(6-7):596-600.

3. Law GW, Lin D, Thomas R. Colonic phytobezoar as a rare cause of large bowel obstruction. BMJ Case Rep. 2015;2015.

4. Agha FP, Nostrant TT, Fiddian-Green RG. "Giant colonic bezoar:" a medication bezoar due to psyllium seed husks.
Am J Gastroenterol. 1984;79(4):319-321.

5. Potyk D. Intestinal obstruction from impacted antacid tablets. N Engl J Med. 1970;283(3):134-135.

6. Ripolles T, Garcia-Aguayo J, Martinez MJ, Gil P. Gastrointestinal bezoars: sonographic and CT characteristics. AJR Am J Roentgenol. 2001;177(1):65-69. 